\title{
Lithium Electrochemistry of WS 2 Nanoflakes Studied by In-situ TEM
}

\author{
Yaobin $\mathrm{Xu}^{1,2}$, Joohoon $\mathrm{Kang}^{2}$, Mark C. Hersam ${ }^{2}$, Jinsong $\mathrm{Wu}^{1,2}$, Vinayak P. Dravid ${ }^{1,2}$ \\ 1. NUANCE Center, Northwestern University, Evanston, IL, United States \\ 2. Department of Materials Science and Engineering, Northwestern University, Evanston, IL, United \\ States
}

Layered tungsten disulfide $\left(\mathrm{WS}_{2}\right)$ shows a unique combination of valuable structural, electronic, optical, mechanical, chemical, and thermal properties that have been studied for decades [1]. The large interlayer separation in $\mathrm{WS}_{2}(\sim 0.65 \mathrm{~nm})$ provides perfect locations to accommodate guest species such as alkali metal ions $\left(\mathrm{Li}^{+}, \mathrm{Na}^{+}\right.$, and $\left.\mathrm{K}^{+}\right)$through a process known as intercalation. Intercalation in layer-structured materials such as $\mathrm{MoS}_{2}$ have been extensively studied for applications in energy storage [2-4]. Unlike $\mathrm{MoS}_{2}, \mathrm{WS}_{2}$ has large lattice parameters and smaller bandgap, which indicates that its better electrochemistry performance but current research lacks. Recent developments of in-situ transmission electron microscopy (TEM), as one unique tool to conduct real time structural measurements under the dynamic electrochemical reaction processes [5]. Such in-operando measurements make it possible to analyze and tackle the intricacies of the lithiation mechanism in electrode materials during charge/discharge cycles. Herein, we applied both the in-situ electron diffraction with a remarkably low electron beam dosage and atomic resolution TEM (HRTEM) images to view along the same crystallographic direction and study the dynamic structural evolutions of lithium intercalated $\mathrm{WS}_{2}$ nanoflakes during lithiation. The electron diffraction patterns is then carefully compared to the HRTEM results to identify the beam effect on $\mathrm{WS}_{2}$ nanoflakes. It is to study the dynamic and structural evolutions and unravel the mechanism of $\mathrm{WS}_{2}$ lithiation.

Figure 1 shows the pristine state (a-c) and lithiated (d-f) $\mathrm{WS}_{2}$ nanoflakes. Electron diffraction pattern of the pristine $\mathrm{WS}_{2}$ indicates a nature of perfect single crystal. After lithiation, the $\mathrm{WS}_{2}$ nanoflake is converted to $\mathrm{Li}_{2} \mathrm{~S}$ and $\mathrm{W}$ nanoparticles. In addition, there is also an in-plane expansion around $16.6 \%$. As shown in Figure 2 (a-f), the time-lapse electron diffraction patterns (EDPs) of $\mathrm{WS}_{2}$ nanoflake acquired along the [001] zone-axis after the reaction time of 0,120, 210, 220, 240, 300 seconds, respectively. The [001] zone axis EDP of the pristine $2 \mathrm{H}-\mathrm{WS}_{2}$ (Figure 2(a)) shows one set of 6-fold symmetry diffraction spots. After 120 s of lithiation, the (100) lattice $d$-spacing is gradually increased corresponding to the gradually increase of intercalated lithium. At 210s, surprisingly, the extra spots start to appear as indicated by green arrow. At 220s, the diffraction spots corresponding to a commensurate superstructure become much clearer (Figure 2(e)). At 240s, the newly appeared diffraction spot as indicated by yellow arrow indicates the formation of $\mathrm{Li}_{2} \mathrm{~S}$. Finally, the fully lithiated $\mathrm{WS}_{2}$ nanoflake is converted to $\mathrm{Li}_{2} \mathrm{~S}$ and $\mathrm{W}$ nanoparticles without any lithium-intercalated superstructure (Figure 2(f)). This structural evolution can be described by a two-step reaction: intercalation reaction followed by conversion reaction. A phase transformation from $2 \mathrm{H}-1 \mathrm{~T}$ can be identified at the intercalating stage. Moreover, the EDPs results are coincide with HRTEM results very well, which means $\mathrm{WS}_{2}$ nanoflake is relatively stable under the electron beam irradiation.

The presentation will also cover ongoing investigations and data analysis; all of which may provide further insights into the understanding of the lithiation kinetics and mechanisms in typical layered transition-metal dichalcogenide structures. We believe these will be helpful in designing next generation advanced lithium ion batteries [6]. 


\section{References:}

[1] Q.H. Wang, et al, Nat. Nanotechnol. 7 (2012), p. 699.

[2] L. David, R. Bhandavat and G. Singh, ACS Nano 8 (2014), p. 1759.

[3] L.F. Wang, et al, J. Am. Chem. Soc. 136 (2014), p. 6693.

[4] Q.Q. Li, et al, Nano Energy 38 (2017), p. 342.

[5] J.Y. Huang, et al, Science 330 (2010), p. 1515.

[6] This work was supported by the NUANCE Center at Northwestern University, using the EPIC facility that receives support from the Soft and Hybrid Nanotechnology Experimental (SHyNE) Resource (NSF NNCI1542205); the MRSEC program (NSF DMR-1121262) at the Materials Research Center; the International Institute for Nanotechnology (IIN); the Keck Foundation; and the State of Illinois, through the IIN.
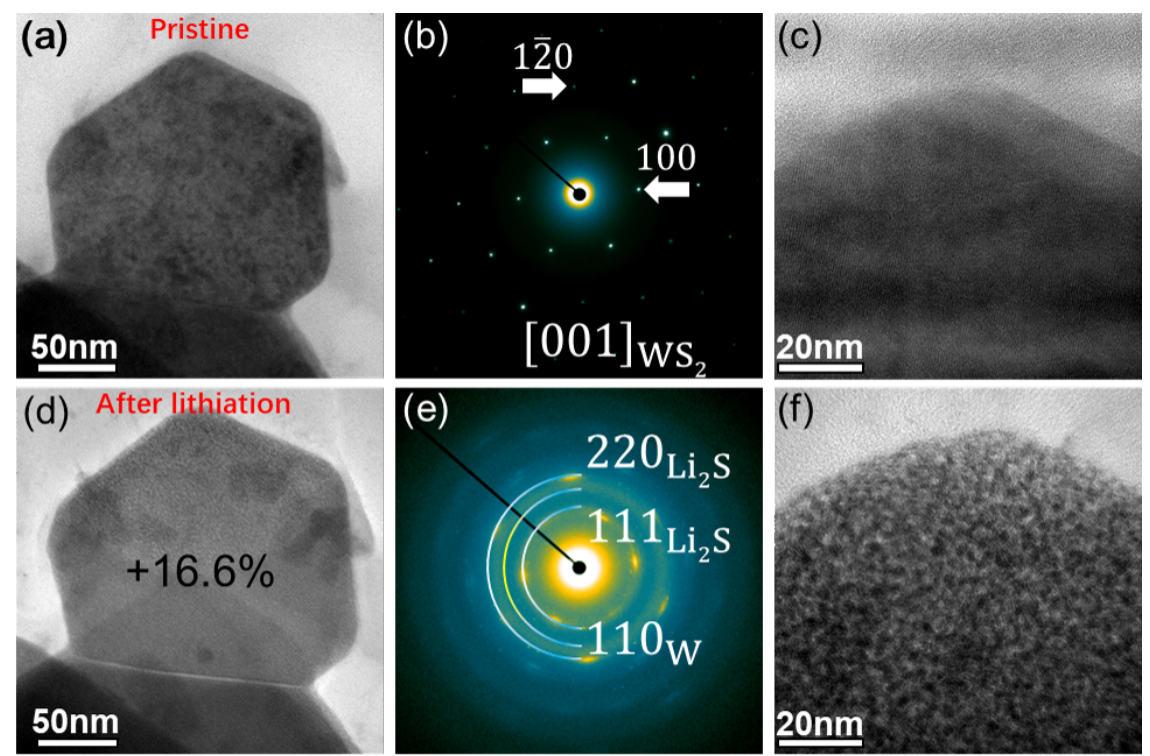

Figure 1. Lithiation of $\mathrm{WS}_{2}$. (a-c) Pristine state of $\mathrm{WS}_{2}$ nanoflake. (d-f) Lithiated $\mathrm{WS}_{2}$.
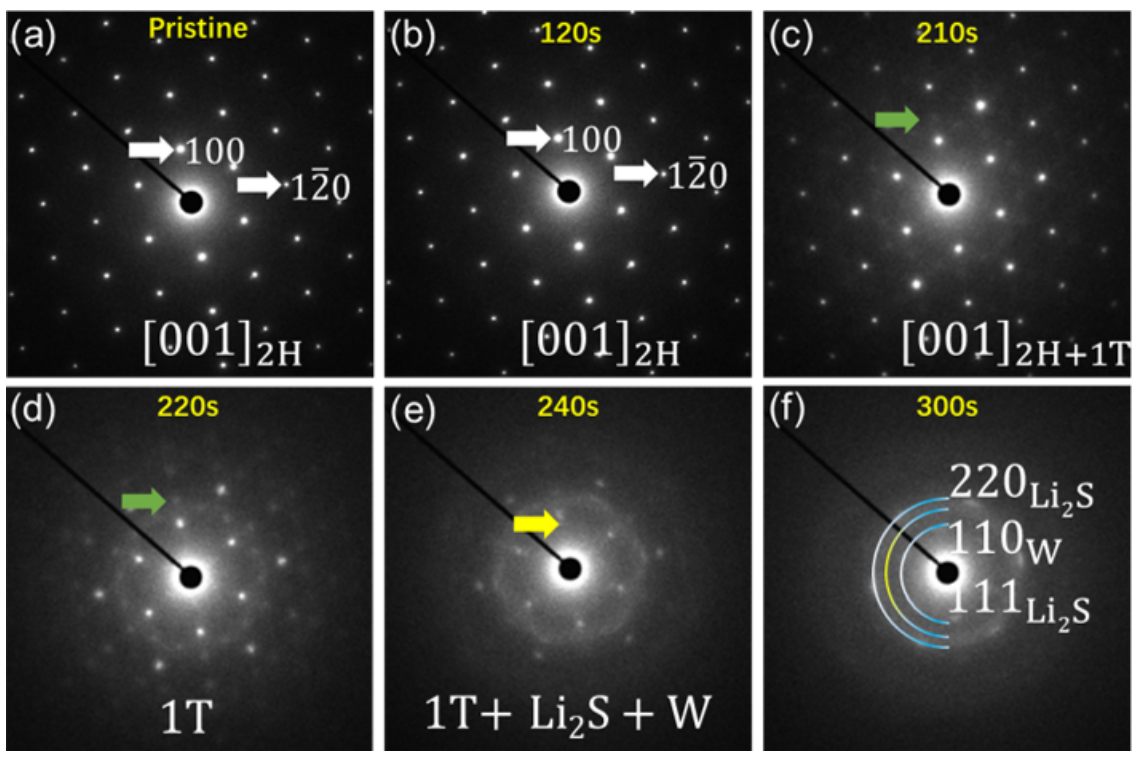

Figure 2. In-situ electron diffraction of $\mathrm{WS}_{2}$ lithiation. (a-f) In-situ electron diffraction patterns (EDPs) along the [001] zone-axis during the lithiation process. 